\title{
Enabling Extensive Numerical Problem Solving on Smartphones and Tablets
}

\section{Dr. Michael B. Cutlip, University of Connecticut}

Michael B. Cutlip is an Emeritus Professor within the Chemical and Biomolecular Engineering Department at the University of Connecticut and has served as department head and director of the university's Honors Program. He has B. Ch. E. and M. S. degrees from Ohio State and a Ph. D. from the University of Colorado. He has been the Chair and National Program Chair for the ASEE Chemical Engineering Division plus he co-chaired the ASEE Summer School for Chemical Engineering faculty in 2002. His current interests include the development of general software for numerical problem solving and application to chemical and biochemical engineering. Dr. Cutlip is also managing director of Polymath Software that develops and provides problem solving software to higher educational institutions and to individual professional and academics users.

\section{Dr. Mordechai Shacham, Ben-Gurion University of the Negev}

Mordechai Shacham is professor emeritus of the Department of Chemical Engineering at the Ben-Gurion University of the Negev in Israel. He has served as department head, as the chairman of the Israeli Inter-University Center for e-Learning (IUCEL) and as the president of the Israel Institute of Chemical Engineers (IIChE). He is the recipient of the of the 2000 CACHE Award, the 2010 Himmelblau Award of the CAST division of the AIChE and he is a Fellow of the IIChE. Dr. Shacham received his BSc and DSc degrees from the Technion, Israel Institute of Technology. His research interests include analysis, modeling and regression of data, applied numerical methods and prediction and consistency analysis of physical properties.

\section{Michael Elly , Ben-Gurion University of the Negev}

Michael Elly holds BSc and MBA degrees from the Ben-Gurion University of the Negev in Israel. He is with the Intel Corporation that he joined in 1996 and has served in several senior IT/Automation positions in Israel and in the US. He is the lead programmer for PolyMath and the new PolyMathLite software packages. He is also currently pursuing a Ph. D. in Chemical Engineering at the Ben-Gurion University. 


\section{Enabling Extensive Numerical Problem Solving on Smartphones and Tablets}

\section{Introduction}

Problem solving is an essential part of engineering education, and many educational benefits can be derived from solving realistic problems. There is an emerging field of Simulation-Based Engineering Science, SBES, which will positively affect "discovery, health, energy, environment, security, and quality of life for all peoples."(1) There are many types of problem solving involved in SBES; however, the simulation and solution of real engineering problems is very important and often requires the application of numerical methods. This is one area of problem solving that sets the engineering students and engineering professionals apart from many other disciplines. Thus the advancement of engineering education worldwide and particularly in developing countries requires resources for the efficient application of numerical methods by engineering students, faculty and staff.

Access to software and the computing resources that can enable numerical problem solving is a particular challenge to engineering departments in developing countries as often desktop computers and interactive terminals within computers labs are very limited or unavailable. Educational use of numerical analysis within engineering coursework and laboratories while desirable is often difficult to achieve.

The authors of this paper have devoted the last 30 years to the continuing development of Polymath Software ${ }^{(2)}$ that has been developed over the years from a mainframe package under the Plato Educational computer system marketed by the Control Data Corporation to a widely used PC product. The current PolyMath PC software is used by over 120 academic institutions world-wide and is available for purchase via the internet with low-priced educational versions.

While this PC product is continuing to evolve in capabilities, our work has also been expanded into the Android OS that is employed in over 750 million smartphone, tablet and computing devices worldwide. We have recently been successful in creating an Android app called PolyMathLite that provides most of the problem solving capabilities of PolyMath for PCs. This app only requires Android 2.2 or higher so it will execute on even the older smartphones and tablets. PolyMathLite implements the same numerical algorithms and provides similar options to those in Polymath for PCs. Android devices generate comparable solutions to seven significant figures when compared to PC solutions. The calculational capabilities and execution speed of Android phones and tablets are extremely fast. Problem setup and execution is completely on the device so that no active internet connection is needed once the app is installed.

PolyMathLite solves systems of linear equations (LEs), nonlinear algebraic equations (NLEs), systems of ordinary differential equations (ODEs) and carries out polynomial, multiple linear and nonlinear regressions of data (REGs). This software also provides for automatic creation of MATLAB m-files and also automatically creates Excel spreadsheets through the use of Polymath for the PC.

This paper will first consider some future prospects for smartphones, and then consider the implications for engineering education with a focus on developing countries. 
Mobile Smartphones Are Coming to the World

As engineering educators consider the future, particularly in developing countries, there are some emerging tools that can provide assistance with educational calculations and thus with the delivery of e-learning materials. A very exciting tool for engineering calculations is the smartphone that is rapidly taking over the mobile phone marketplace worldwide.

Consider that the smartphone will be the preference for engineering students and engineering professionals. The eMarketer ${ }^{(3)}$, a leading market research company, suggests that "smartphone users worldwide will total 1.75 billion in 2014." eMarketer "expects 4.55 billion people worldwide to use a mobile phone in 2014."

Horace Dediu, founder of Asymco, who covers the mobile industry has made some predictions $^{(4)}$ regarding the future of smartphones. A particularly interesting graphic is reproduced as Figure 1. Note that the smartphone numbers seem to be approaching the world's population in the next 10 years. This is a challenge to consider, but it does indicate that smartphones will probably be available as a possible educational tool with increasing popularity with younger persons as educators look toward the future.

Special Considerations for Africa

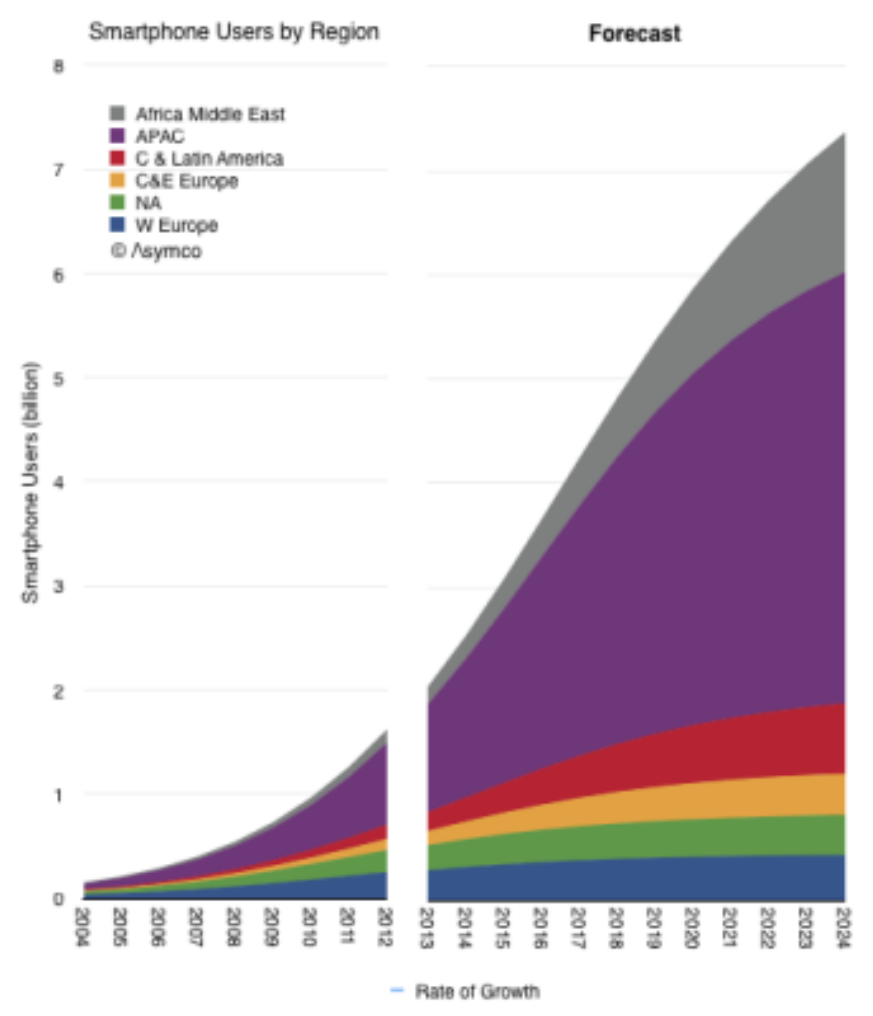

Figure 1 - Projection of Smartphone Users for 10 Years by World Region from H. Dediu ${ }^{(4)}$

Matthew Labrooy, who works with eLearning Africa ${ }^{(5)}$, has written about Africa's smartphone revolution ${ }^{(6)}$. He has estimated that "Smartphone growth in Africa has increased by $43 \%$ every year since 2000, and experts predict that $69 \%$ of mobiles in Africa will have Internet access by 2014. As a result, smartphone manufacturers are showing increased interest in the region in a bid to gain their share of half a billion potential customers. Currently a simple budget smartphone produced for the African market is available on the streets of Kenya for as little as €37 (US\$50) and has sold more than 300,000 units across the country. Smartphone penetration in Africa is estimated between $17-19 \%$, though rates vary wildly from country to country. Nigeria, Africa's most populous nation, has a smartphone penetration as high as $41 \%$." Spurred by the success of the African budget smartphone, Microsoft has produced a smartphone (Windows Phone 8) specifically for the African Market, the Huawei 4Afrika ${ }^{(7)}$, which was released in South Africa, Egypt, Nigeria, Kenya, Ivory Coast, Angola, and Morocco in February 2013. 
There are calculations involving one or several explicit algebraic equations which must be carried out frequently for different parameter values. PML enables setting up the system of equations, solving it for one set of parameter values and saving it for future use. The problem can be reloaded, whenever necessary, and resolved for different sets of parameter values.

Consider for example a problem involving the calculation of constant annual payment $(P m t)$ and future value $(F n)$ for a loan with amount of $P$, to be repaid in n years with interest rate of $i$. The parameters of this problem are: $P, n$ and $i$. Consider $P=1000$, $n=25$ and $i=10 \%$. This problem can then be entered into PML as shown in phone Display 1 along with the on-display entry keyboard.

Note that the \# sign indicates a user comment section. The text in the first line must contain a comment which is used for the problem identification and reload when a problem is saved. Except in the first line, PML disregards the text inserted after the \# sign. The constant definitions and the explicit equations can be entered in any order as PML reorders the equations according to the computational sequence. The problem is solved by a tap on the "Run" button. If there are errors in the problem code syntax, then feedback is given along with the line numbers.

When the problem is entered correctly, the PML report that contains the variable values at the solution is presented as shown in Display 2. In this particular case the solution is: $P m t=\$ 110$ and $F n=\$ 10800$. The problem can be saved with the title of "\# Constant annual payment" and rerun with different parameter values whenever necessary.

Most of the intrinsic functions, if... then...else statements, and logical variables available to computer programming software are available within PML.

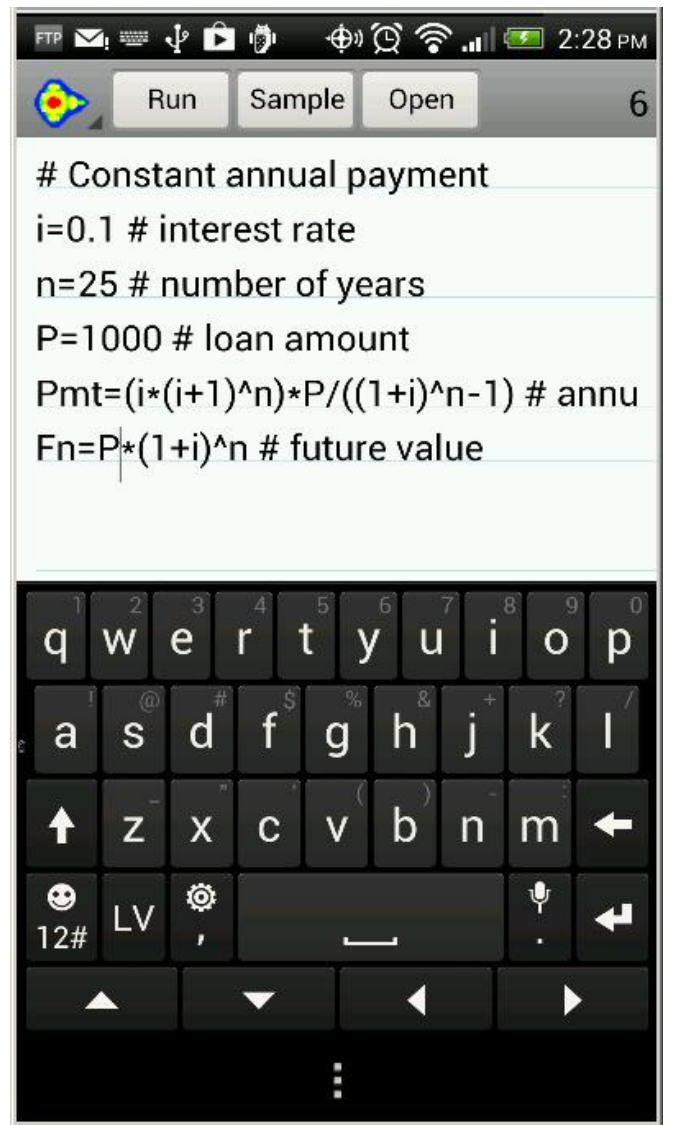

Display 1

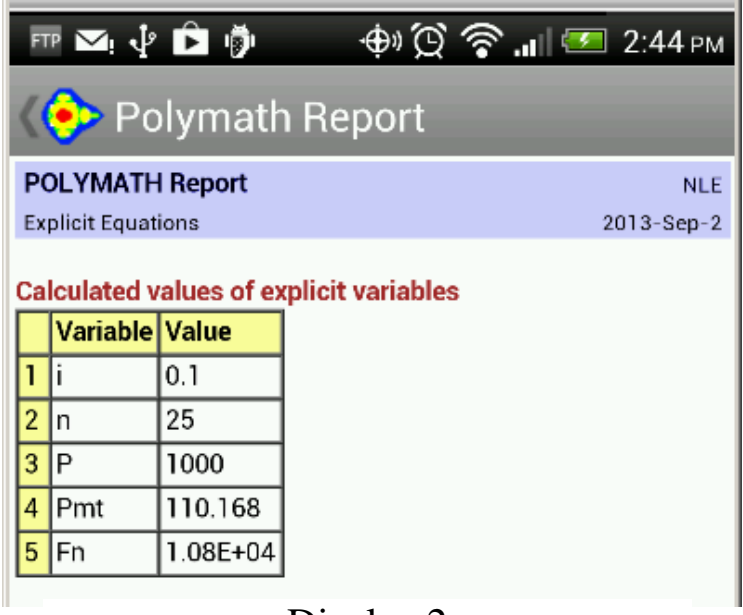

Display 2 
Fitting Lines, Curves, and Equations to Data

Computations involving the fitting of a straight line, a polynomial or a complex mathematical model to data (regression) are very widely used in many disciplines. The textbook of Montgomery and Runger ${ }^{(8)}$ contains examples involving regressions from the disciplines of biology, chemistry, physics, economics, medicine, sports and chemical, civil, mechanical, electrical, industrial, materials, energy and environmental engineering.

Linear Regression - A medical related example presented by Montgomery and Runger ${ }^{(8)}$ involves the fitting of a linear regression model to the relationship between hypertension (blood pressure rise in mm of mercury, $y$ ) the noise level (in decibels, $x)$. The problem can be specified in PML as shown in Display 3.

The $\mathbf{x}$ and $\mathbf{y}$ data are entered in an array format (only part of the $\mathbf{x}$ data are shown). The command "polyfit $\mathrm{x}, \mathrm{y}, 1$ " specifies that a $1^{\text {st }}$ degree polynomial (straight line) is sought. The solution report for the problem (Display 4) provides the numerical values of the model parameters $\mathrm{a}_{0}$ and $\mathrm{a}_{1}$, the statistical measures for the goodness of the fit: the correlation coefficient $\left(R^{2}\right)$, the confidence intervals and the variance. The solution report includes, also, a table containing the observed (measured) $\mathbf{y}$ values, the predicted $\mathbf{y}$ values ( $\left.\mathrm{y}_{\text {calc }}\right)$ and the residuals (Delta $\left.\mathrm{y}=\mathrm{y}-\mathrm{y}_{\text {calc }}\right)$.

A quick analysis of the adequacy of the linear model to represent the data can be obtained by visual inspection of the plot of $y$ and ycalc versus $x$ in Figure 2 on the next page. The data for such a plot containing the
Display 3
\# Relationship between noise exposure (x)

\# and hypertension (y)

\# $y=a 0+a 1^{*} x$

\# Verified solution $a 0=-10.1315$, a1 $=0.174294$

$x=[60,63,65,70,70,70,80,90,80,80,85,89,90,90,90,90,94$

$y=[1,0,1,2,5,1,4,6,2,3,5,4,6,8,4,5,7,9,7,6]$

polyfit $x, y, 1$

values of the dependent and independent variables can be exported to plotting utilities. We have been using the Plotim Android application for this purpose. Plotim Free Graphs and Plotim Graph Maker are Android applications available from Google Play. The plot demonstrates, indeed, that the blood pressure rise increases linearly with the increase of the noise level. 


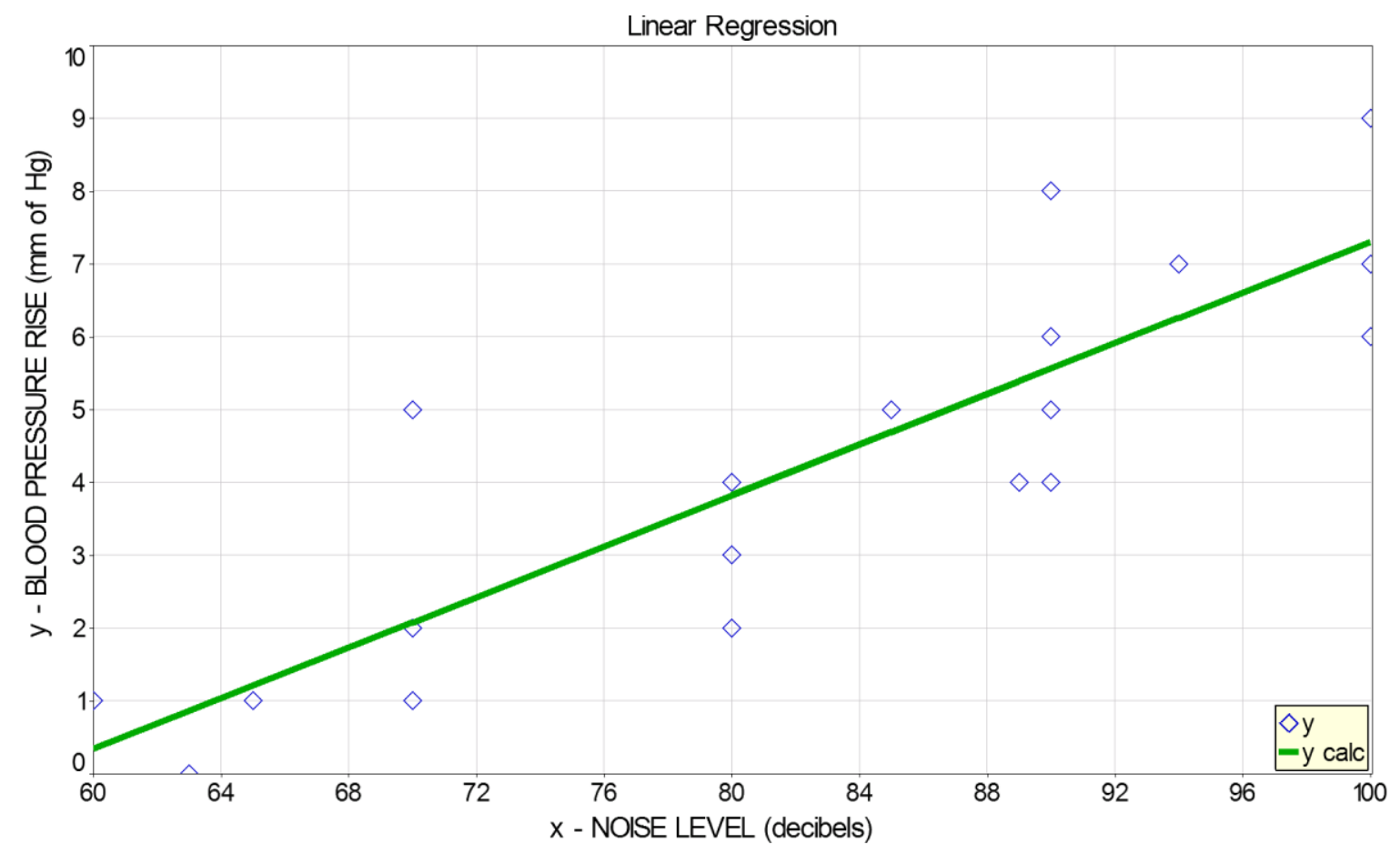

Figure 2 - Blood Pressure Rise versus Noise Level ${ }^{(7)}$

\section{Modeling Dynamic Behavior with Differential Equations}

Differential equations (in particular ordinary differential equations, ODE's) are used for modeling dynamics of processes. One such model, frequently used in ecological studies, is the predator-prey model. The model determines the population over time of the two different species given parameters relating to the interaction between them. Consider for example the version presented by Wolfram demonstrations ${ }^{(9)}$.

In this example the predators are foxes (with population: $F$ ) and the preys are rabbits (with population: $R$ ). Rabbits, which live on vegetation, grow at a rate proportional to the current population: $a R$ and die from encounters with Foxes given by $-\alpha R F$. Foxes grow at a rate proportional to the encounters with rabbits, $\beta R F$, and die at a rate proportional to the current population, $-c F$. The problem definition as entered into PML (including the parameter values and initial and final values of the variables) is presented in Display 5. Note that the differential equation syntax is very similar to the

\# Predator-Prey Model $d(R) / d(t)=a * R$-alpha* $R^{*} F$ \# Balance on Rabbit Population $R(0)=1$ \# Initial Condition of Rabbit Population $d(F) / d(t)=$ beta* $R * F-b * F$ \# Balance on Fox Population $F(0)=0.5$ \# Initial Condition of Fox Population \#Current Parameter Values :

$a=1$

alpha $=0.5$

$\mathrm{b}=0.75$

beta $=0.25$

$t(0)=0$ \# Initial Condition for Independent Variable $t(f)=15$ \# Final Value for Independent Variable mathematical form.

Display 5 
The solution to the predator/prey problem is plotted in Display 6 using the Plotim Android application. This plots the variation of the rabbit and fox population versus time. Observe that the population profiles of the rabbits $(R)$ and the foxes $(F)$ for the solution are cyclic and out of phase with each other.

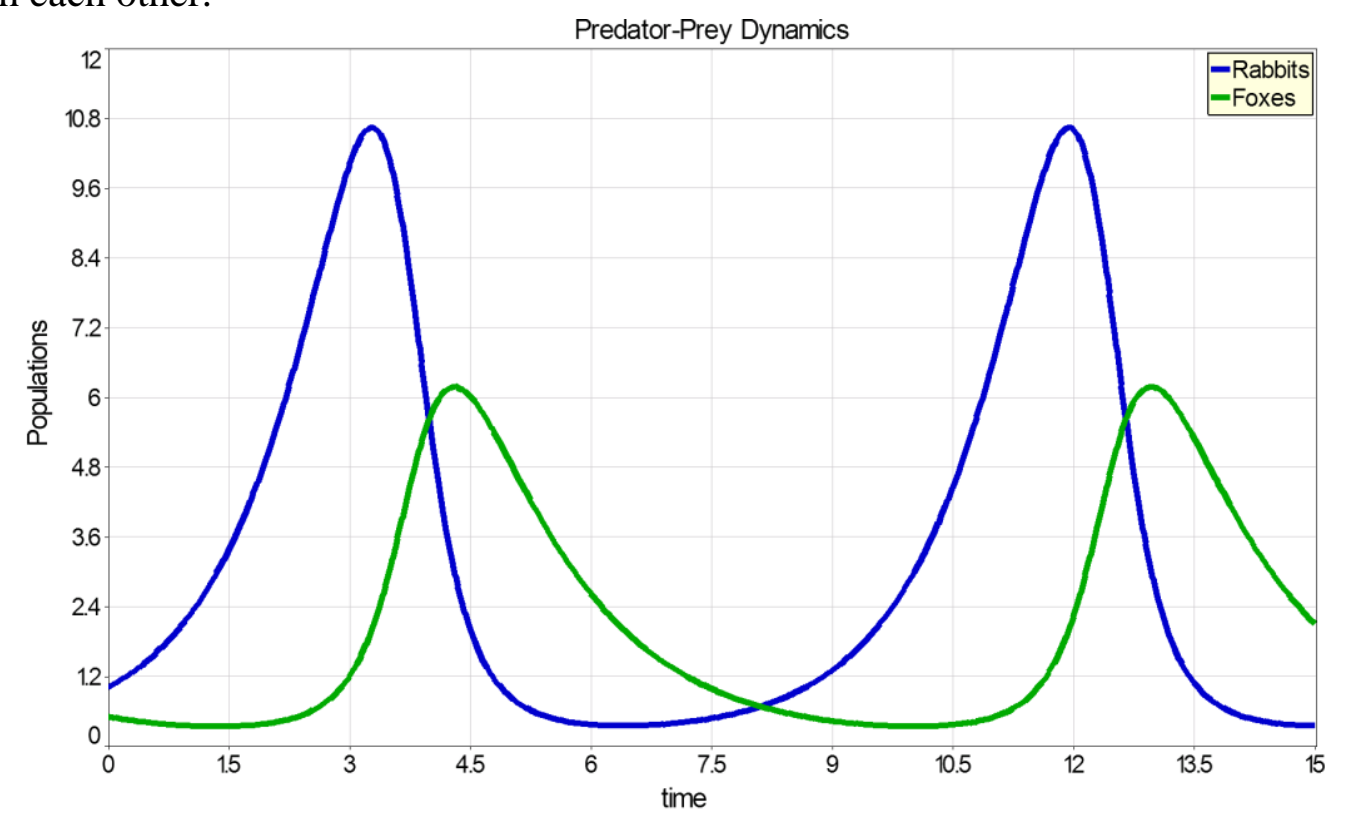

\section{Solving Systems of Nonlinear Equations}

Consider a simple problem that involves finding the intersection of the two curves $y_{1}$ $=\operatorname{sqrt}\left(4-\mathrm{x}^{2}\right)$ and $y_{2}=\mathrm{x}^{3}$ within the first quadrant. Noting that at the intersection $y_{1}=$ $y_{2} \equiv y$ the equations can be rewritten in implicit forms: $f_{1}(x, y)=\mathrm{f}(\mathrm{x})=x^{2}+y^{2}-4=0$ and $f_{2}(x, y)=\mathrm{f}(\mathrm{y})=y-x^{3}=0$. This is shown graphically in Figure 3. The PML solution code is shown in Display 7. Initial estimates for the values $x$ and $y$ should be specified to be in the first quadrant. The solution is found in the "Polymath Report" shown in Display 8. The results presented include the values of $x$ and $y$ at the intersection point, and the values $f_{1}(x, y)$ and $f_{2}(x, y)$ are very close to zero at the solution.

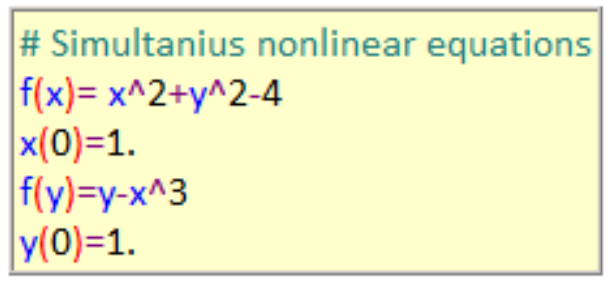

Display 7
Display 6

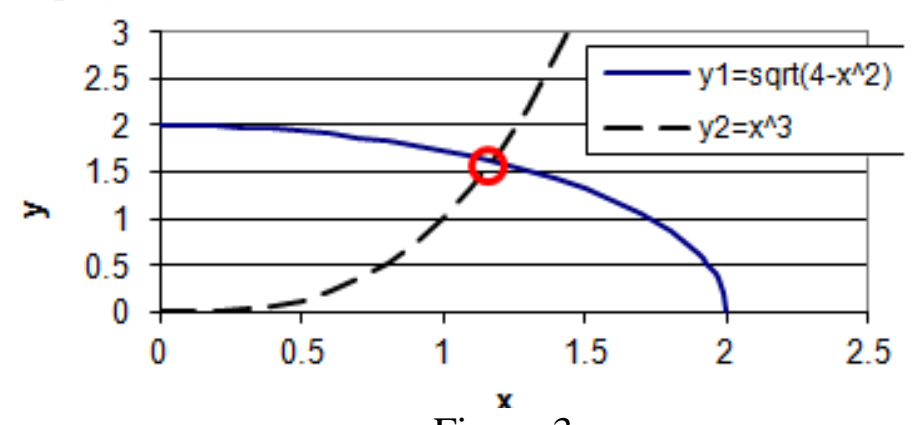

Figure 3
POLYMATH Report

Nonlinear Equations

2013-

Calculated values of NLE variables

\begin{tabular}{|l|l|l|l|l|}
\hline & Variable & Value & $\mathbf{f}(\mathbf{x})$ & Initial Guess \\
\hline 1 & $\mathrm{x}$ & 1.17422 & $4.7 \mathrm{E}-10$ & 1 \\
\hline 2 & $\mathrm{y}$ & 1.61901 & $-1.6 \mathrm{E}-09$ & 1 \\
\hline
\end{tabular}

Nonlinear equations

$$
\begin{aligned}
& 1 f(x)=x^{\wedge} 2+y^{\wedge} 2-4=0 \\
& 2 f(y)=y-x^{\wedge} 3=0
\end{aligned}
$$

Display 8 
Utilization Modes of PolyMath Software when Computing Resources are Scarce

PolyMath PC Educational Site Licenses - PolyMath is currently available to academic institutions with an inexpensive site license that can be provided to students, faculty and staff via computer labs and the institutional secure computer networks. Institutions, colleges and departments can purchase according to their needs. Users can use the PC version directly or can use PolyMathLite on their phone or tablet. Note that coded problems that run on the Android device are interchangeable with those on the PC version and vice versa. Let us assume that the computer labs and networks have Microsoft Excel and MATLAB available.

The PML user with an Android device can develop, enter, solve and save problems. All these activities are done completely on the device and do not require an internet or active phone connection. The Run option automatically generates a Polymath Report that contains the entire problem description including the complete solution, the PolyMath problem

POLYMATH Report
Nonlinear Equations
Calculated values of NLE variables
\begin{tabular}{|l|l|l|l|l|l|} 
2014-Mar-18 \\
\hline & Variable & Value & $\mathbf{f ( x )}$ & Initial Guess & Initial $\mathbf{f ( x )}$ \\
\hline 1 & $\mathrm{x}$ & 1.17422 & $4.7 \mathrm{E}-10$ & 1 & $-2.0 \mathrm{E} 00$ \\
\hline 2 & $\mathrm{y}$ & 1.61901 & $-1.6 \mathrm{E}-09$ & 1 & $0.0 \mathrm{E} 00$ \\
\hline
\end{tabular}
source text, and a MATLAB m-file of the solution. There are then PML options to send this PolyMath Report via 1) Bluetooth, 2) Gmail or 3) another mail package.

The options to send the PolyMath Report via email enables the PML user to send it to his or her own self as an easy way to take this file to a $\mathrm{PC}$ or to the computer network within the institution's network. Once the file is on the PC or the network, the PML user can easily copy the problem source text into the PolyMath PC software for further use including enhanced output, printing or incorporation into reports or presentations.

An interesting option once the problem is in a $\mathrm{PC}$ is to copy the $\mathrm{m}$-file to a MATLAB editor either in the PC or on a computer network in order to execute the program. This allows the user to further modify the m-file to take advantage of advanced MATLAB capabilities. Another option via the PC would be to export the PolyMath program to Microsoft Excel by using the option to automatically create a

Nonlinear equations
$\begin{aligned} & 1 \mathrm{f}(\mathrm{x})=\mathrm{x}^{\wedge} 2+\mathrm{y}^{\wedge} 2-4=0 \\ & 2 \mathrm{f}(\mathrm{y})=\mathrm{y}-\mathrm{x}^{\wedge} 3=0\end{aligned}$

Problem source text

$\#$ Simultanius nonlinear equations
$f(x)=x^{\wedge} 2+y^{\wedge} 2-4$
$x(0)=1$.
$f(y)=y-x^{\wedge} 3$
$y(0)=1$.

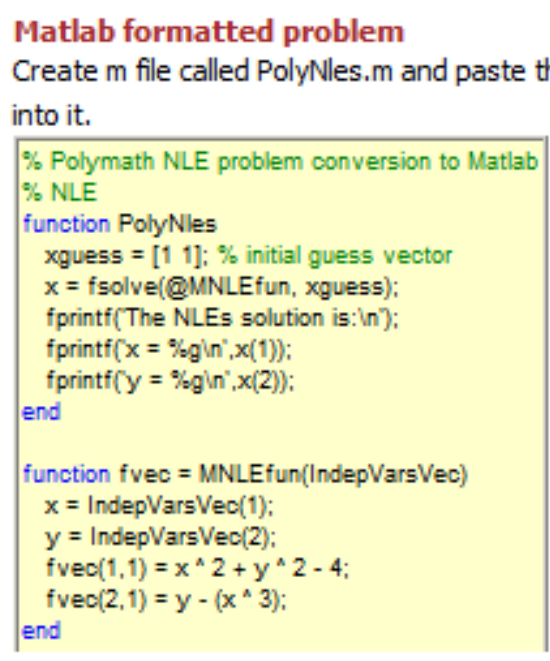
working spreadsheet. Many other possibilities are available to work groups of students including sharing work via Bluetooth, using one group phone as a mobile hotspot, and allowing group members to communicate with other computers and networks via Wi-Fi. Many other options exist for group work and interactions including active learning in class and e-Learning activities. 


\section{Application to Engineering Education}

Engineering disciplines have both general and individual needs for numerical problem solving in various curricula. In chemical engineering, publications by Cutlip et al. ${ }^{(10,11)}$ provide many examples that can be used for instructional purposes.

Engineering educators should begin to consider educational materials that can also incorporate more modeling and numerical problem solving. Smartphones will provide inexpensive and convenient calculational devices for student use. They can also become important delivery devices for e-learning, software packages and educational modules. Smartphones will become very important, particularly in developing countries, as they become more widely available.

\section{Conclusions}

An application for numerical solution of problems (PolyMathLite ${ }^{(12)}$, PML), for Android-based smartphones and tablets, has been developed. This paper and other work have demonstrated with many documented examples that this app is able to efficiently utilize numerical methods to:

- solve problems represented by sets of explicit linear equations

- solve systems of nonlinear algebraic equations that may include variable constraints

- identify the parameter values and calculate statistical metrics for linear, multiple linear, polynomial, and general nonlinear regression problems

- solve stiff and non-stiff systems of ordinary differential equations

- provide solution results in numerical, tabular and graphical formats that allow for clear presentation and rapid interpretation

\section{Bibliography}

(1) Oden, J. T., et al., "Simulation-Based Engineering Science - Revolutionizing Engineering Science through Simulation," Report of the National Science Foundation (USA), Arlington, VA (2006) http://www.nsf.gov/pubs/reports/sbes_final_report.pdf

(2) http://www.polymath-software.com/

(3) http://www.emarketer.com/Article/Smartphone-Users-Worldwide-Will-Total-175-Billion-2014/1010536

(4) http://www.asymco.com/2014/01/07/when-will-smartphones-saturate/

(5) http://www.elearning-africa.com/eLA Newsportal/africas-smartphone-revolution/

(6) http://www.elearning-africa.com/

(7) http://www.microsoft.com/africa/4afrika/

(8) Montgomery, D. C. and G. C. Runger, Applied Statistics and Probability for Engineers, $5^{\text {th }}$ Ed., John Wiley (2011)

(9) http://demonstrations.wolfram.com/PredatorPreyModel/

(10) Cutlip, M. B., Hwalek, J. J., Nuttall, H. E., Shacham, M., Brule, J., Widman, J., Han, T., Finlayson, B., Rosen, E. M. and R. Taylor, "A Collection of Ten Numerical Problems in Chemical Engineering Solved by Various Mathematical Software Packages," Comput. Appl. Eng. Educ., 6(3), 169-180 (1998)

(11) Cutlip, M. B. and Shacham, M., Problem Solving In Chemical and Biochemical Engineering with Polymath, Excel and MATLAB, Prentice-Hall, Upper Saddle River, New-Jersey, 2008

(12) http://www.polymathlite.com/ 